\title{
Euler Matrices and their Algebraic Properties Revisited
}

\author{
Yamilet Quintana ${ }^{1, *}$, William Ramírez ${ }^{2}$ and Alejandro Urieles ${ }^{3}$ \\ ${ }^{1}$ Department of Pure and Applied Mathematics, Postal Code: 89000, Caracas 1080 A, Simon Bolívar University, Venezuela \\ ${ }^{2}$ Department of Natural and Exact Sciences, University of the Coast - CUC, Barranquilla, Colombia \\ ${ }^{3}$ Faculty of Basic Sciences - Mathematics Program, University of Atlántico, Km 7, Port Colombia, Barranquilla, Colombia
}

Received: 2 Sep. 2019, Revised: 23 Feb. 2020, Accepted: 13 Apr. 2020

Published online: 1 Jul. 2020

\begin{abstract}
This paper addresses the generalized Euler polynomial matrix $\mathscr{E}^{(\alpha)}(x)$ and the Euler matrix $\mathscr{E}$. Taking into account some properties of Euler polynomials and numbers, we deduce product formulae for $\mathscr{E}^{(\alpha)}(x)$ and define the inverse matrix of $\mathscr{E}$. We establish some explicit expressions for the Euler polynomial matrix $\mathscr{E}(x)$, which involves the generalized Pascal, Fibonacci and Lucas matrices, respectively. From these formulae, we get some new interesting identities involving Fibonacci and Lucas numbers. Also, we provide some factorizations of the Euler polynomial matrix in terms of Stirling matrices, as well as a connection between the shifted Euler matrices and Vandermonde matrices.
\end{abstract}

Keywords: Euler polynomials, Euler matrix, generalized Euler matrix, generalized Pascal matrix, Fibonacci matrix, Lucas matrix.

\section{Introduction}

The classical Euler polynomials $E_{n}(x)$ and the generalized Euler polynomials $E_{n}^{(\alpha)}(x)$ of (real or complex) order $\alpha$, are usually defined, as follows (see, for details, [1, 16,21, 23]):

$$
\left(\frac{2}{e^{z}+1}\right)^{\alpha} e^{x z}=\sum_{n=0}^{\infty} E_{n}^{(\alpha)}(x) \frac{z^{n}}{n !}, \quad|z|<\pi, \quad 1^{\alpha}:=1,
$$

and

$$
E_{n}(x):=E_{n}^{(1)}(x), \quad n \in \mathbb{N}_{0},
$$

where $\mathbb{N}_{0}:=\mathbb{N} \cup\{0\}$ and $\mathbb{N}=\{1,2,3, \ldots\}$.

The numbers $E_{n}^{(\alpha)}:=E_{n}^{(\alpha)}(0)$ are called generalized Euler numbers of order $\alpha$. The classical Euler numbers $\varepsilon_{n}$ are defined by the generating function

$$
\frac{2}{e^{z}+e^{-z}}=\sum_{n=0}^{\infty} \varepsilon_{n} \frac{z^{n}}{n !}
$$

From (1)-(3), it is easy to check that the connection between the classical Euler numbers and the Euler polynomials is given by the formula

$$
\varepsilon_{n}=2^{n} E_{n}\left(\frac{1}{2}\right), \quad n \in \mathbb{N}_{0} .
$$

Thus, the numbers $E_{n}:=E_{n}(0)$ are also known in pieces of literature as Euler numbers (cf., e.g., [15,21]). The first six generalized Euler polynomials are

$$
\begin{aligned}
E_{0}^{(\alpha)}(x)= & 1, \quad E_{1}^{(\alpha)}(x)=x-\frac{\alpha}{2}, \\
E_{2}^{(\alpha)}(x)= & x^{2}-\alpha x+\frac{\alpha(\alpha-1)}{4}, \\
E_{3}^{(\alpha)}(x)= & x^{3}-\frac{3 \alpha}{2} x^{2}+\frac{3 \alpha(\alpha-1)}{4} x-\frac{\alpha^{2}(\alpha-3)}{8}, \\
E_{4}^{(\alpha)}(x)= & x^{4}-2 \alpha x^{3}+\frac{3 \alpha(\alpha-1)}{2} x^{2}-\frac{\alpha^{2}(\alpha-3)}{2} x \\
& +\frac{\alpha(\alpha-1)\left(\alpha^{2}-5 \alpha-2\right)}{16}, \\
E_{5}^{(\alpha)}(x)= & x^{5}-\frac{5 \alpha}{2} x^{4}+\frac{5 \alpha(\alpha-1)}{2} x^{3}-\frac{5 \alpha^{2}(\alpha-3)}{4} x^{2} \\
& +\frac{5 \alpha(\alpha-1)\left(\alpha^{2}-5 \alpha-2\right)}{16} x \\
& -\frac{\alpha^{2}\left(\alpha^{3}-10 \alpha^{2}+15 \alpha+10\right)}{32} .
\end{aligned}
$$

For a broad information on old literature and new research trends on these classes of polynomials, we strongly encourage the interested reader to $[5,8,9,10,15$, $16,17,18,19,20,21,22,24,25,26,27]$.

*Corresponding author e-mail: yquintana@usb.ve 
From the generating relation (1), it is fairly straightforward to deduce the addition formula:

$$
E_{n}^{(\alpha+\beta)}(x+y)=\sum_{k=0}^{n}\left(\begin{array}{l}
n \\
k
\end{array}\right) E_{k}^{(\alpha)}(x) E_{n-k}^{(\beta)}(y) .
$$

And, it follows that

$$
E_{n}^{(\alpha)}(x+1)+E_{n}^{(\alpha)}(x)=2 E_{n}^{(\alpha-1)}(x) .
$$

Since $E_{n}^{(0)}(x)=x^{n}$, making the substitution $\beta=0$ into (5) and interchanging $x$ and $y$, we get

$$
E_{n}^{(\alpha)}(x+y)=\sum_{k=0}^{n}\left(\begin{array}{l}
n \\
k
\end{array}\right) E_{k}^{(\alpha)}(y) x^{n-k} .
$$

As an immediate consequence, we have

$$
\begin{gathered}
E_{n}(x+y)=\sum_{k=0}^{n}\left(\begin{array}{l}
n \\
k
\end{array}\right) E_{k}(y) x^{n-k}, \\
E_{n}(x)=\sum_{k=0}^{n}\left(\begin{array}{l}
n \\
k
\end{array}\right) E_{k} x^{n-k} .
\end{gathered}
$$

Using (4), (8) and the well known relation $E_{n}(1-x)=(-1)^{n} E_{n}(x)$, it is possible to deduce the following connection formula between $E_{n}$ and the classical Euler numbers $\varepsilon_{n}$ :

$$
E_{n}=\left\{\begin{array}{l}
-\frac{1}{2^{n}} \sum_{k=0}^{n}\left(\begin{array}{l}
n \\
k
\end{array}\right) \varepsilon_{n-k}, \quad \text { if } n \text { is odd } \\
0, \quad \text { if } n \text { is even. }
\end{array}\right.
$$

Inspired by the article [32] in which the authors introduce the generalized Bernoulli matrix and show some of its algebraic properties, we examine some properties of generalized Euler matrix.

The outline of the paper is, as follows: Section 2 has an auxiliary character and provides some background as well as some results which will be used throughout the paper. In Section 3, we introduce the generalized Euler matrix and investigate some interesting particular cases of this matrix, namely the Euler polynomial matrix, the Euler matrix, and the specialized Euler matrix. The main results of this section are Theorems 1,2, 3 and 4, which contain the information concerning the product formula for the Euler matrix, an explicit expression for the inverse matrix of the specialized Euler matrix, the factorization of the Euler matrix via the generalized Pascal matrix of first kind, and a useful factorization for the inverse matrix of a particular "horizontal sliding" of the Euler polynomial matrix, respectively. Section 4 shows some factorizations of the generalized Euler matrix in terms the Fibonacci and Lucas matrices, respectively (cf. Theorems 5 and 6). Also, some new identities involving Fibonacci and Lucas numbers are presented in this section. In Section 5, we provide some factorizations of the Euler polynomial matrix in terms of Stirling matrices, and the shifted Euler matrices as well as their connection with Vandermonde matrices. Section 6 is devoted to conclusion and further research.

\section{Background and previous results}

Throughout this paper, all matrices are in $M_{n+1}(\mathbb{R})$, the set of all $(n+1)$-square matrices over the real field. Also, for $i, j$ any nonnegative integers, we adopt the following convention

$$
\left(\begin{array}{l}
i \\
j
\end{array}\right)=0, \text { whenever } j>i \text {. }
$$

In this section, we recall the definitions of the generalized Pascal matrix, Fibonacci matrix and, Lucas matrix.

Definition 1.Let $x$ be any nonzero real number. The generalized Pascal matrix of first type $P[x] \in M_{n+1}(\mathbb{R})$ is the matrix whose entries are given by (see [2, 29]):

$$
p_{i, j}(x)=\left\{\begin{array}{l}
\left(\begin{array}{l}
i \\
j
\end{array}\right) x^{i-j}, \quad i \geq j, \\
0, \quad \text { otherwise. }
\end{array}\right.
$$

In [2,29,31], some properties of the generalized Pascal matrix of first type are shown, for example, its matrix factorization by special summation matrices, its associated differential equation and its bivariate extensions. The following proposition summarizes some algebraic and differential properties of $P[x]$.

Proposition 1.Let $P[x] \in M_{n+1}(\mathbb{R})$ be the generalized Pascal matrix of first type. Then, the following statements hold.

(a)Special value. If the convention $0^{0}=1$ is adopted, then it is possible to define

$$
P[0]:=I_{n+1}=\operatorname{diag}(1,1, \ldots, 1),
$$

where $I_{n+1}$ denotes the identity matrix of order $n+1$. (b) $P[x]$ is an invertible matrix and its inverse is given by

$$
P^{-1}[x]:=(P[x])^{-1}=P[-x] .
$$

(c) [2, Theorem 2] Addition theorem of the argument. For $x, y \in \mathbb{R}$, we have

$$
P[x+y]=P[x] P[y] .
$$

(d)[2, Theorem 5] Differential relation (Appell type polynomial entries). $P[x]$ satisfies the following differential equation

$$
D_{x} P[x]=\mathfrak{L} P[x]=P[x] \mathfrak{L},
$$

where $D_{x} P[x]$ is the matrix resulting from taking the derivative with respect to $x$ of each entry of $P[x]$ and the entries of the $(n+1) \times(n+1)$ matrix $\mathfrak{L}$ are given by

$1_{i, j}=\left\{\begin{array}{l}p_{i, j}^{\prime}(0), \quad i \geq j, \\ 0, \quad \text { otherwise, }\end{array}=\left\{\begin{array}{l}j+1, \quad i=j+1, \\ 0, \quad \text { otherwise. }\end{array}\right.\right.$ 
(e)([29, Theorem 1]) The matrix $P[x]$ can be factorized, as follows:

$$
P[x]=G_{n}[x] G_{n-1}[x] \cdots G_{1}[x],
$$

where $G_{k}[x]$ is the $(n+1) \times(n+1)$ summation matrix given by

$$
G_{k}[x]=\left\{\begin{array}{l}
{\left[\begin{array}{cc}
I_{n-k} & 0 \\
0 & S_{k}[x]
\end{array}\right], \quad k=1, \ldots, n-1,} \\
S_{n}[x], \quad k=n,
\end{array}\right.
$$

being $S_{k}[x]$ the $(k+1) \times(k+1)$ matrix whose entries $S_{k}(x ; i, j)$ are given by

$$
S_{k}(x ; i, j)=\left\{\begin{array}{l}
x^{i-j}, \quad j \leq i, \quad(0 \leq i, j \leq k) . \\
0, \quad j>i,
\end{array}\right.
$$

Another necessary structured matrices in what follows, are the Fibonacci and Lucas matrices. Below, we recall the definitions of each matrix.

Definition 2.Let $\left\{F_{n}\right\}_{n \geq 1}$ be the Fibonacci sequence, i.e., $F_{n}=F_{n-1}+F_{n-2}$ for $n \geq 2$ with initial conditions $F_{0}=0$ and $F_{1}=1$. The Fibonacci matrix $\mathscr{F} \in M_{n+1}(\mathbb{R})$ is the matrix whose entries are given by [13]:

$$
f_{i, j}=\left\{\begin{array}{l}
F_{i-j+1}, \quad i-j+1 \geq 0, \\
0, \quad i-j+1<0 .
\end{array}\right.
$$

Let $\mathscr{F}^{-1}$ be the inverse of $\mathscr{F}$ and denote by $\tilde{f}_{i, j}$ the entries of $\mathscr{F}^{-1}$. In [13], the authors obtained the following explicit expression for $\mathscr{F}^{-1}$.

$$
\tilde{f}_{i, j}=\left\{\begin{array}{l}
1, \quad i=j, \\
-1, \quad i=j+1, j+2, \\
0, \quad \text { otherwise. }
\end{array}\right.
$$

Definition 3.Let $\left\{L_{n}\right\}_{n \geq 1}$ be the Lucas sequence, i.e. $L_{n+2}=L_{n+1}+L_{n}$ for $n \geq 1$ with initial conditions $L_{1}=1$ and $L_{2}=3$. The Lucas matrix $\mathscr{L} \in M_{n+1}(\mathbb{R})$ is the matrix whose entries are given by [33]:

$$
l_{i, j}=\left\{\begin{array}{l}
L_{i-j+1}, \quad i-j \geq 0, \\
0, \quad \text { otherwise. }
\end{array}\right.
$$

Let $\mathscr{L}^{-1}$ be the inverse of $\mathscr{L}$ and denote by $\tilde{l}_{i, j}$ the entries of $\mathscr{L}^{-1}$. In [33, Theorem 2.2], the authors obtained the following explicit expression for $\mathscr{L}^{-1}$.

$$
\tilde{l}_{i, j}=\left\{\begin{array}{l}
1, \quad i=j, \\
-3, \quad i=j+1, \\
5(-1)^{i-j} 2^{i-j-2}, \quad i \geq j+2, \\
0, \quad \text { otherwise. }
\end{array}\right.
$$

For $x$ any nonzero real number, the following relation between the matrices $P[x]$ and $\mathscr{L}$ was stated and proved in [33, Theorem 3.1].

$$
P[x]=\mathscr{L} \mathscr{G}[x]=\mathscr{H}[x] \mathscr{L},
$$

where the entries of the $(n+1) \times(n+1)$ matrices $\mathscr{G}[x]$ and $\mathscr{H}[x]$ are given by

$$
\begin{aligned}
g_{i, j}(x)= & x^{-j-1}\left[x^{i+1}\left(\begin{array}{c}
i \\
j
\end{array}\right)-3 x^{i}\left(\begin{array}{c}
i-1 \\
j
\end{array}\right)\right] \\
& +x^{-j-1}\left[5(-1)^{i+1} 2^{i-1} m_{i-1, j+1}\left(\frac{x}{2}\right)\right], \\
h_{i, j}(x)= & x^{-j-1}\left[x^{i+1}\left(\begin{array}{c}
i \\
j
\end{array}\right)-3 x^{i}\left(\begin{array}{c}
i \\
j+1
\end{array}\right)\right. \\
& +x^{-j-1}\left[(-1)^{j+1} \frac{5 x^{i+j+2}}{2^{j+3}} n_{i+1, j+3}\left(\frac{2}{x}\right)\right],
\end{aligned}
$$

respectively, with

$$
\begin{aligned}
m_{i, j}(x):=\left\{\begin{array}{l}
\sum_{k=j}^{i}(-1)^{k}\left(\begin{array}{l}
k \\
j
\end{array}\right) x^{k}, \quad i \geq j, \\
0, \quad i<j,
\end{array}\right. \\
n_{i, j}(x):=\left\{\begin{array}{l}
\sum_{k=j}^{i}(-1)^{k}\left(\begin{array}{l}
i \\
k
\end{array}\right) x^{k}, \quad i \geq j, \\
0, \quad i<j .
\end{array}\right.
\end{aligned}
$$

and

\section{The generalized Euler matrix}

Definition 4.The generalized $(n+1) \times(n+1)$ Euler matrix $\mathscr{E}^{(\alpha)}(x)$ is defined by

$$
E_{i, j}^{(\alpha)}(x)=\left\{\begin{array}{l}
\left(\begin{array}{c}
i \\
j
\end{array}\right) E_{i-j}^{(\alpha)}(x), \quad i \geq j \\
0, \quad \text { otherwise }
\end{array}\right.
$$

While, $\mathscr{E}(x):=\mathscr{E}^{(1)}(x)$ and $\mathscr{E}:=\mathscr{E}(0)$ are called the Euler polynomial matrix and the Euler matrix, respectively. In the particular case $x=\frac{1}{2}$, we call the matrix $\mathbb{E}:=\mathscr{E}\left(\frac{1}{2}\right)$ specialized Euler matrix.

It is clear that (6) yields the following matrix identity:

$$
\mathscr{E}^{(\alpha)}(x+1)+\mathscr{E}^{(\alpha)}(x)=2 \mathscr{E}^{(\alpha-1)}(x) .
$$

Since $\mathscr{E}^{(0)}(x)=P[x]$, replacing $\alpha$ by 1 in (15), we have

$$
\mathscr{E}(x+1)+\mathscr{E}(x)=2 P[x] .
$$

Then, putting $x=0$ in (16) and taking into account (11), we get

$$
\mathscr{E}(1)+\mathscr{E}=2 I_{n+1} .
$$


Analogously,

$$
\mathscr{E}+\mathscr{E}(-1)=2 P[-1]
$$

From (4), it follows that the entries of the specialized Euler matrix $\mathbb{E}$ are given by

$$
e_{i, j}=\left\{\begin{array}{l}
\left(\begin{array}{l}
i \\
j
\end{array}\right) 2^{j-i} \varepsilon_{i-j}, \quad i \geq j, \\
0, \quad \text { otherwise. }
\end{array}\right.
$$

From (10), it follows that the entries of the Euler matrix $\mathscr{E}$ are given by

$$
E_{i, j}=\left\{\begin{array}{l}
\left(\begin{array}{l}
i \\
j
\end{array}\right) E_{i-j}, \quad i>j \text { and } i-j \text { odd }, \\
1, \quad i=j, \\
0, \quad \text { otherwise. }
\end{array}\right.
$$

The next result is an immediate consequence of Definition 4 and the addition formula (5).

Theorem 1.The generalized Euler matrix $\mathscr{E}^{(\alpha)}(x)$ satisfies the following product formula.

$$
\begin{aligned}
\mathscr{E}^{(\alpha+\beta)}(x+y)= & \mathscr{E}^{(\alpha)}(x) \mathscr{E}^{(\beta)}(y)=\mathscr{E}^{(\beta)}(x) \mathscr{E}^{(\alpha)}(y) \\
& =\mathscr{E}^{(\alpha)}(y) \mathscr{E}^{(\beta)}(x) .
\end{aligned}
$$

Proof.We proceed as in the proof of [32, Theorem 2.1], making the corresponding modifications. Let $A_{i, j}^{(\alpha, \beta)}(x, y)$ be the $(i, j)$-th entry of the matrix product $\mathscr{E}^{(\alpha)}(x) \mathscr{E}^{(\beta)}(y)$. Then, by the addition formula (5), we have

$$
\begin{aligned}
A_{i, j}^{(\alpha, \beta)}(x, y) & =\sum_{k=0}^{n}\left(\begin{array}{l}
i \\
k
\end{array}\right) E_{i-k}^{(\alpha)}(x)\left(\begin{array}{c}
k \\
j
\end{array}\right) E_{k-j}^{(\beta)}(y) \\
& =\sum_{k=j}^{i}\left(\begin{array}{l}
i \\
k
\end{array}\right) E_{i-k}^{(\alpha)}(x)\left(\begin{array}{c}
k \\
j
\end{array}\right) E_{k-j}^{(\beta)}(y) \\
& =\sum_{k=j}^{i}\left(\begin{array}{c}
i \\
j
\end{array}\right)\left(\begin{array}{c}
i-j \\
i-k
\end{array}\right) E_{i-k}^{(\alpha)}(x) E_{k-j}^{(\beta)}(y) \\
& =\left(\begin{array}{c}
i \\
j
\end{array}\right) \sum_{k=0}^{i-j}\left(\begin{array}{c}
i-j \\
k
\end{array}\right) E_{i-j-k}^{(\alpha)}(x) E_{k}^{(\beta)}(y) \\
& =\left(\begin{array}{c}
i \\
j
\end{array}\right) E_{i-j}^{(\alpha+\beta)}(x+y),
\end{aligned}
$$

which implies the first equality of (18). The second and third equalities of (18) can be derived in a similar way.

Corollary 1.Let $\left(x_{1}, \ldots, x_{k}\right) \in \mathbb{R}^{k}$. For $\alpha_{j}$ real or complex parameters, the Euler matrix $\mathscr{E}^{\left(\alpha_{j}\right)}(x)$ satisfies the following product formula, $j=1, \ldots, k$.

$$
\mathscr{E}^{\left(\alpha_{1}+\alpha_{2}+\cdots+\alpha_{k}\right)}\left(x_{1}+x_{2}+\cdots+x_{k}\right)=\prod_{j=1}^{k} \mathscr{E}^{\left(\alpha_{j}\right)}\left(x_{j}\right) .
$$

Proof.The application of induction on $k$ gives the desired result.

Taking $x=x_{1}=x_{2}=\cdots=x_{k}$ and $\alpha=\alpha_{1}=\alpha_{2}=\cdots=$ $\alpha_{k}$, we obtain the following simple formula for the powers of the generalized Euler matrix, and consequently, for the powers of the Euler polynomial and Euler matrices.

Corollary 2.The generalized Euler matrix $\mathscr{E}^{(\alpha)}(x)$ satisfies the following identity.

$$
\left(\mathscr{E}^{(\alpha)}(x)\right)^{k}=\mathscr{E}^{(k \alpha)}(k x) .
$$

In particular,

$$
\begin{aligned}
(\mathscr{E}(x))^{k} & =\mathscr{E}^{(k)}(k x), \\
\mathscr{E}^{k} & =\mathscr{E}^{(k)} .
\end{aligned}
$$

Remark.Note that Theorem 1 and Corollaries 1 and 2 are respectively, the analogous of Theorem 2.1 and Corollaries 2.2 and 2.3 of [32] in the setting of Euler matrices.

Let $\mathscr{D} \in M_{n+1}(\mathbb{R})$ be the matrix whose entries are defined by

$$
d_{i, j}=\left\{\begin{array}{l}
\left(1+(-1)^{i-j}\right)\left(\begin{array}{l}
i \\
j
\end{array}\right) 2^{j-i-1}, \quad i \geq j, \\
0, \quad \text { otherwise. }
\end{array}\right.
$$

Theorem 2.The inverse matrix of the specialized Euler matrix $\mathbb{E}$ is given by

$$
\mathbb{E}^{-1}=\mathscr{D}
$$

Furthermore,

$$
\left[\mathscr{E}^{(k)}\left(\frac{k}{2}\right)\right]^{-1}=\mathscr{D}^{k} .
$$

Proof.Taking into account (4) and (17), it is possible to deduce

$$
\begin{aligned}
& \sum_{k=0}^{n} \frac{\left(1+(-1)^{k}\right)}{2}\left(\begin{array}{l}
n \\
k
\end{array}\right) 2^{n-k} E_{n-k}\left(\frac{1}{2}\right) \\
& =\sum_{k=0}^{n} \frac{\left(1+(-1)^{k}\right)}{2}\left(\begin{array}{l}
n \\
k
\end{array}\right) \varepsilon_{n-k}=\delta_{n, 0},
\end{aligned}
$$

where $\delta_{n, 0}$ is the Kronecker delta (cf., e.g., [19, pp. 107-109]). Hence, we obtain that the $(i, j)$-th entry of the matrix product $\mathscr{D} \mathbb{E}$ may be written as

$$
\begin{aligned}
& \sum_{k=j}^{i}\left(\begin{array}{l}
i \\
k
\end{array}\right) \frac{\left(1+(-1)^{i-k}\right)}{2} 2^{k-i}\left(\begin{array}{c}
k \\
j
\end{array}\right) E_{k-j}\left(\frac{1}{2}\right) \\
= & \left(\begin{array}{c}
i \\
j
\end{array}\right) 2^{j-i} \sum_{k=j}^{i}\left(\begin{array}{c}
i-j \\
k-j
\end{array}\right) \frac{\left(1+(-1)^{i-k}\right)}{2} 2^{k-j} E_{k-j}\left(\frac{1}{2}\right) \\
= & \left(\begin{array}{c}
i \\
j
\end{array}\right) 2^{j-i} \sum_{k=0}^{i-j}\left(\begin{array}{c}
i-j \\
k
\end{array}\right) \frac{\left(1+(-1)^{i-j-k}\right)}{2} 2^{k} E_{k}\left(\frac{1}{2}\right) \\
= & \left(\begin{array}{l}
i \\
j
\end{array}\right) 2^{j-i} \delta_{i-j, 0},
\end{aligned}
$$


and consequently, $\mathscr{D} \mathbb{E}=I_{n+1}$. Similar arguments allow to show that $\mathbb{E} \mathscr{D}=I_{n+1}$, so $\mathbb{E}^{-1}=\mathscr{D}$. that

Finally, from the identity $\mathbb{E}^{-1}=\mathscr{D}$ and (19) we see

$$
\left[\mathscr{E}^{(k)}\left(\frac{k}{2}\right)\right]^{-1}=\left(\mathbb{E}^{k}\right)^{-1}=\left(\mathbb{E}^{-1}\right)^{k}=\mathscr{D}^{k}
$$

This last chain of equalities finishes the proof.

The calculation of $\mathbb{E}^{-1}$ depends on the use of inverse relations derived from exponential generating functions (cf. [19, Chap. 3, Sec. 3.4]). This tool can be applied to define $\mathbb{E}^{-1}$, but it does not work for determining of $\mathscr{E}^{-1}$. This fact and (10) suggest that methodology proposed in [32] does not suffice to find an explicit formula for $\mathscr{E}^{-1}$.

The next result establishes the relation between the generalized Euler matrix and the generalized Pascal matrix of first type.

Theorem 3.The generalized Euler matrix $\mathscr{E}^{(\alpha)}(x)$ satisfies the following relation.

$$
\begin{aligned}
\mathscr{E}^{(\alpha)}(x+y) & =\mathscr{E}^{(\alpha)}(x) P[y]=P[x] \mathscr{E}^{(\alpha)}(y) \\
& =\mathscr{E}^{(\alpha)}(y) P[x] .
\end{aligned}
$$

In particular,

$$
\begin{gathered}
\mathscr{E}(x+y)=P[x] \mathscr{E}(y)=P[y] \mathscr{E}(x), \\
\mathscr{E}(x)=P[x] \mathscr{E} \\
\mathscr{E}\left(x+\frac{1}{2}\right)=P[x] \mathbb{E},
\end{gathered}
$$

and

$$
\mathscr{E}=P\left[-\frac{1}{2}\right] \mathbb{E}
$$

Proof. The substitution $\beta=0$ into (18) yields

$$
\begin{aligned}
\mathscr{E}^{(\alpha)}(x+y) & =\mathscr{E}^{(\alpha)}(x) \mathscr{E}^{(0)}(y)=\mathscr{E}^{(0)}(x) \mathscr{E}^{(\alpha)}(y) \\
& =\mathscr{E}^{(\alpha)}(y) \mathscr{E}^{(0)}(x) .
\end{aligned}
$$

Since $\mathscr{E}^{(0)}(x)=P[x]$, we obtain

$$
\mathscr{E}^{(\alpha)}(x+y)=P[x] \mathscr{E}^{(\alpha)}(y) .
$$

A similar argument allows to show that $\mathscr{E}^{(\alpha)}(x+y)=\mathscr{E}^{(\alpha)}(x) P[y]$ and $\mathscr{E}^{(\alpha)}(x+y)=\mathscr{E}^{(\alpha)}(y) P[x]$.

Next, the substitution $\alpha=1$ into (20) yields (21). From the substitutions $y=0$ and $y=\frac{1}{2}$ into (21), we obtain the relations (22) and (23), respectively.

Finally, the substitution $x=-\frac{1}{2}$ into (23) completes the proof.
Remark. Note that the relation (21) is the analogous of [32, Eq. (13)] in the context of Euler polynomial matrices and, the counterpart of (22) is [32, Eq. (14)]. However, the relation (23) is slightly different from [32, Eq. (14)], since it involves an Euler polynomial matrix with "shifted argument" and the specialized Euler matrix. More precisely, the relation [32, Eq. (14)] reads as

$$
\mathscr{B}(x)=P[x] \mathscr{B} .
$$

Consequently, this relation expresses the Bernoulli polynomial matrix $\mathscr{B}(x)$ in terms of the matrix product between the generalized Pascal matrix of first type $P[x]$ and the Bernoulli matrix $\mathscr{B}$. While, on the left hand side of (23), an Euler polynomial matrix with "shifted argument" appears, and the matrix product on the right hand side of (23) contains the specialized Euler matrix $\mathbb{E}$.

The following example shows validity of Theorem 3 .

Example 1.Let us consider $n=3$. It follows from the definition 4 that

$\mathbb{E}=\left[\begin{array}{cccc}1 & 0 & 0 & 0 \\ 0 & 1 & 0 & 0 \\ -\frac{1}{4} & 0 & 1 & 0 \\ 0 & -\frac{3}{4} & 0 & 1\end{array}\right], \mathscr{E}\left(x+\frac{1}{2}\right)=\left[\begin{array}{cccc}1 & 0 & 0 & 0 \\ x & 1 & 0 & 0 \\ x^{2}-\frac{1}{4} & 2 x & 1 & 0 \\ x^{3}-\frac{3}{4} x & 3 x^{2}-\frac{3}{4} & 3 x & 1\end{array}\right]$.

On the other hand, from (23) and a simple computation, we have

$$
\begin{aligned}
\mathscr{E}\left(x+\frac{1}{2}\right) & =\underbrace{\left[\begin{array}{cccc}
1 & 0 & 0 & 0 \\
x & 1 & 0 & 0 \\
x^{2} & 2 x & 1 & 0 \\
x^{3} & 3 x^{2} & 3 x & 1
\end{array}\right]}_{P[x]} \underbrace{\left[\begin{array}{cccc}
1 & 0 & 0 & 0 \\
0 & 1 & 0 & 0 \\
-\frac{1}{4} & 0 & 1 & 0 \\
0 & -\frac{3}{4} & 0 & 1
\end{array}\right]}_{\mathbb{E}} \\
& =\left[\begin{array}{cccc}
1 & 0 & 0 & 0 \\
x & 1 & 0 & 0 \\
x^{2}-\frac{1}{4} & 2 x & 1 & 0 \\
x^{3}-\frac{3}{4} x & 3 x^{2}-\frac{3}{4} & 3 x & 1
\end{array}\right] .
\end{aligned}
$$

The next theorem is followed by a simple computation.

Theorem 4.The inverse of the Euler polynomial matrix $\mathscr{E}\left(x+\frac{1}{2}\right)$ can be expressed as

$$
\left[\mathscr{E}\left(x+\frac{1}{2}\right)\right]^{-1}=\mathbb{E}^{-1} P[-x]=\mathscr{D} P[-x] .
$$

In particular,

$$
\mathscr{E}^{-1}=\mathscr{D} P\left[\frac{1}{2}\right]
$$

Proof.Using (12), (23) and Theorem 2, the relation (24) is deduced. The substitution $x=-\frac{1}{2}$ into (24) yields (25). 
Example 2.Let us consider $n=3$. From the definition 4 and a standard computation, we obtain

$$
\begin{aligned}
{\left[\mathscr{E}\left(x+\frac{1}{2}\right)\right]^{-1} } & \left.=\left[\begin{array}{cccc}
1 & 0 & 0 & 0 \\
x & 1 & 0 & 0 \\
x^{2}-\frac{1}{4} & 2 x & 1 & 0 \\
x^{3}-\frac{3}{4} x & 3 x^{2}-\frac{3}{4} & 3 x & 1
\end{array}\right]\right]^{-1} \\
& =\left[\begin{array}{cccc}
1 & 0 & 0 & 0 \\
-x & 1 & 0 & 0 \\
x^{2}+\frac{1}{4} & -2 x & 1 & 0 \\
-x^{3}-\frac{3}{4} x & 3 x^{2}+\frac{3}{4} & -3 x & 1
\end{array}\right] .
\end{aligned}
$$

On the other hand, from (24), we have

$$
\begin{aligned}
{\left[\mathscr{E}\left(x+\frac{1}{2}\right)\right]^{-1} } & =\underbrace{\left[\begin{array}{llll}
1 & 0 & 0 & 0 \\
0 & 1 & 0 & 0 \\
\frac{1}{4} & 0 & 1 & 0 \\
0 & \frac{3}{4} & 0 & 1
\end{array}\right]}_{\mathscr{D}} \underbrace{\left[\begin{array}{cccc}
1 & 0 & 0 & 0 \\
-x & 1 & 0 & 0 \\
x^{2} & -2 x & 1 & 0 \\
-x^{3} & 3 x^{2} & -3 x & 1
\end{array}\right]}_{P[-x]} \\
& =\left[\begin{array}{cccc}
1 & 0 & 0 & 0 \\
-x & 1 & 0 & 0 \\
x^{2}+\frac{1}{4} & -2 x & 1 & 0 \\
-x^{3}-\frac{3}{4} x & 3 x^{2}+\frac{3}{4} & -3 x & 1
\end{array}\right] .
\end{aligned}
$$

Hence, when $x=-\frac{1}{2}$, we get

$$
\mathscr{E}^{-1}=\underbrace{\left[\begin{array}{llll}
1 & 0 & 0 & 0 \\
0 & 1 & 0 & 0 \\
\frac{1}{4} & 0 & 1 & 0 \\
0 & \frac{3}{4} & 0 & 1
\end{array}\right]}_{\mathscr{D}} \underbrace{\left[\begin{array}{cccc}
1 & 0 & 0 & 0 \\
\frac{1}{2} & 1 & 0 & 0 \\
\frac{1}{4} & 1 & 1 & 0 \\
\frac{1}{8} & \frac{3}{4} & \frac{3}{2} & 1
\end{array}\right]}_{P\left[\frac{1}{2}\right]}=\left[\begin{array}{cccc}
1 & 0 & 0 & 0 \\
\frac{1}{2} & 1 & 0 & 0 \\
\frac{1}{2} & 1 & 1 & 0 \\
\frac{1}{2} & \frac{3}{2} & \frac{3}{2} & 1
\end{array}\right] .
$$

At this point, we should refer to the recent work [11] since it states an explicit formula to the inverse matrix of the $q$-Pascal matrix plus one in terms of the $q$-analogue of the Euler matrix $\mathscr{E}$.

As a consequence of the relations (13), (14), and Theorems 3 and 4, we obtain the following corollaries.

Corollary 3.The Euler polynomial matrix $\mathscr{E}\left(x+\frac{1}{2}\right)$ and its inverse can be factorized by summation matrices, as follows:

$$
\begin{gathered}
\mathscr{E}\left(x+\frac{1}{2}\right)=G_{n}[x] G_{n-1}[x] \cdots G_{1}[x] \mathbb{E} \\
{\left[\mathscr{E}\left(x+\frac{1}{2}\right)\right]^{-1}=\mathscr{D} G_{n}[-x] G_{n-1}[-x] \cdots G_{1}[-x] .}
\end{gathered}
$$

In particular,

$$
\begin{aligned}
\mathscr{E} & =G_{n}\left[-\frac{1}{2}\right] G_{n-1}\left[-\frac{1}{2}\right] \cdots G_{1}\left[-\frac{1}{2}\right] \mathbb{E}, \\
\mathscr{E}^{-1} & =\mathscr{D} G_{n}\left[\frac{1}{2}\right] G_{n-1}\left[\frac{1}{2}\right] \cdots G_{1}\left[\frac{1}{2}\right] .
\end{aligned}
$$

Corollary 4.For $x$ any nonzero real number, the Euler polynomial matrix $\mathscr{E}\left(x+\frac{1}{2}\right)$ and its inverse can be factorized, respectively, in terms of the Lucas matrix $\mathscr{L}$ and its inverse, as follows:

$$
\begin{gathered}
\mathscr{E}\left(x+\frac{1}{2}\right)=\mathscr{L} \mathscr{G}[x] \mathbb{E}=\mathscr{H}[x] \mathscr{L} \mathbb{E} \\
{\left[\mathscr{E}\left(x+\frac{1}{2}\right)\right]^{-1}=\mathscr{D}(\mathscr{G}[x])^{-1} \mathscr{L}^{-1}=\mathscr{D} \mathscr{L}^{-1}(\mathscr{H}[x])^{-1} .}
\end{gathered}
$$

In particular,

$$
\begin{aligned}
\mathscr{E} & =\mathscr{L} \mathscr{G}\left[-\frac{1}{2}\right] \mathbb{E}=\mathscr{H}\left[-\frac{1}{2}\right] \mathscr{L} \mathbb{E}, \\
\mathscr{E}^{-1} & =\mathscr{D}\left(\mathscr{G}\left[-\frac{1}{2}\right]\right)^{-1} \mathscr{L}^{-1}=\mathscr{D} \mathscr{L}^{-1}\left(\mathscr{H}\left[-\frac{1}{2}\right]\right)^{-1} .
\end{aligned}
$$

We end this section showing other identities, which can be easily deduced from the content of this paper. Thus, we will omit the details of their proofs.

$$
\begin{aligned}
D_{x} \mathscr{E}(x+y) & =\mathfrak{L} P[x] \mathscr{E}(y), \\
D_{x} \mathscr{E}(x) & =\mathfrak{L} P[x] \mathscr{E} \\
D_{x} \mathscr{E}\left(x+\frac{1}{2}\right) & =\mathfrak{L} P[x] \mathbb{E} \\
D_{x}\left[\mathscr{E}\left(x+\frac{1}{2}\right)\right]^{-1} & =\mathscr{D} \mathfrak{L} P[-x] .
\end{aligned}
$$

\section{Generalized Euler polynomial matrices via Fibonacci and Lucas matrices}

For $0 \leq i, j \leq n$ and $\alpha$ a real or complex number, let $\mathscr{M}^{(\alpha)}(x)$ be the $(n+1) \times(n+1)$ matrix whose entries are given by (cf. [32, Eq. (18)]):

$$
\begin{aligned}
\tilde{m}_{i, j}^{(\alpha)}(x)= & \left(\begin{array}{c}
i \\
j
\end{array}\right) E_{i-j}^{(\alpha)}(x)-\left(\begin{array}{c}
i-1 \\
j
\end{array}\right) E_{i-j-1}^{(\alpha)}(x) \\
& -\left(\begin{array}{c}
i-2 \\
j
\end{array}\right) E_{i-j-2}^{(\alpha)}(x) .
\end{aligned}
$$

We denote $\mathscr{M}(x)=\mathscr{M}^{(1)}(x)$ and $\mathscr{M}=\mathscr{M}(0)$.

Similarly, let $\mathscr{N}^{(\alpha)}(x)$ be the $(n+1) \times(n+1)$ matrix whose entries are given by (cf. [32, Eq. (32)]):

$$
\begin{aligned}
\tilde{n}_{i, j}^{(\alpha)}(x)= & \left(\begin{array}{c}
i \\
j
\end{array}\right) E_{i-j}^{(\alpha)}(x)-\left(\begin{array}{c}
i \\
j+1
\end{array}\right) E_{i-j-1}^{(\alpha)}(x) \\
& -\left(\begin{array}{c}
i \\
j+2
\end{array}\right) E_{i-j-2}^{(\alpha)}(x) .
\end{aligned}
$$


We denote $\mathscr{N}(x)=\mathscr{N}^{(1)}(x)$ and $\mathscr{N}=\mathscr{N}(0)$. that

From the definitions of $\mathscr{M}^{(\alpha)}(x)$ and $\mathscr{N}^{(\alpha)}(x)$, we see

$$
\begin{aligned}
& \tilde{m}_{0,0}^{(\alpha)}(x)=\tilde{m}_{1,1}^{(\alpha)}(x)=\tilde{n}_{0,0}^{(\alpha)}(x)=\tilde{n}_{1,1}^{(\alpha)}(x)=E_{0}^{(\alpha)}(x)=1 \\
& \tilde{m}_{0, j}^{(\alpha)}(x)=\tilde{n}_{0, j}^{(\alpha)}(x)=0, \quad j \geq 1 \\
& \tilde{m}_{1,0}^{(\alpha)}(x)=\tilde{n}_{1,0}^{(\alpha)}(x)=E_{1}^{(\alpha)}(x)-E_{0}^{(\alpha)}(x)=x-\frac{\alpha}{2}-1 \\
& \tilde{m}_{1, j}^{(\alpha)}(x)=\tilde{n}_{1, j}^{(\alpha)}(x)=0, \quad j \geq 2 \\
& \tilde{m}_{i, 0}^{(\alpha)}(x)=E_{i}^{(\alpha)}(x)-E_{i-1}^{(\alpha)}(x)-E_{i-2}^{(\alpha)}(x), \quad i \geq 2, \\
& \tilde{n}_{i, 0}^{(\alpha)}(x)=E_{i}^{(\alpha)}(x)-i E_{i-1}^{(\alpha)}(x)-\frac{i(i-1)}{2} E_{i-2}^{(\alpha)}(x), \quad i \geq 2
\end{aligned}
$$

For $0 \leq i, j \leq n$ and $\alpha$ a real or complex number, let $\mathscr{L}_{1}^{(\alpha)}(x)$ be the $(n+1) \times(n+1)$ matrix whose entries are given by

$$
\begin{aligned}
\hat{l}_{i, j}^{(\alpha, 1)}(x) & =\left(\begin{array}{c}
i \\
j
\end{array}\right) E_{i-j}^{(\alpha)}(x)-3\left(\begin{array}{c}
i-j \\
j
\end{array}\right) E_{i-j-1}^{(\alpha)}(x) \\
& =5 \sum_{k=j}^{i-2}(-1)^{i-k} 2^{i-k-2}\left(\begin{array}{c}
k \\
j
\end{array}\right) E_{k-j}^{(\alpha)}(x) .
\end{aligned}
$$

We denote $\mathscr{L}_{1}(x)=\mathscr{L}_{1}^{(1)}(x)$ and $\mathscr{L}_{1}=\mathscr{L}_{1}(0)$.

Similarly, let $\mathscr{L}_{2}^{(\alpha)}(x)$ be the $(n+1) \times(n+1)$ matrix whose entries are given by

$$
\begin{aligned}
\hat{l}_{i, j}^{(\alpha, 2)}(x)= & \left(\begin{array}{c}
i \\
j
\end{array}\right) E_{i-j}^{(\alpha)}(x)-3\left(\begin{array}{c}
i \\
j+1
\end{array}\right) E_{i-j-1}^{(\alpha)}(x) \\
& +5 \sum_{k=j+1}^{i}(-1)^{k-j} 2^{k-j-2}\left(\begin{array}{l}
i \\
k
\end{array}\right) E_{i-k}^{(\alpha)}(x) .
\end{aligned}
$$

We denote $\mathscr{L}_{2}(x)=\mathscr{L}_{2}^{(1)}(x)$ and $\mathscr{L}_{2}=\mathscr{L}_{2}(0)$. that

From the definitions of $\mathscr{L}_{1}^{(\alpha)}(x)$ and $\mathscr{L}_{2}^{(\alpha)}(x)$, we see

$$
\begin{gathered}
\hat{l}_{i, i}^{(\alpha, 1)}(x)=\hat{l}_{i, i}^{(\alpha, 2)}(x)=1, \quad i \geq 0, \\
\hat{l}_{0, j}^{(\alpha, 1)}(x)=\hat{l}_{0, j}^{(\alpha, 2)}(x)=0, \quad j \geq 1, \\
\hat{l}_{1,0}^{(\alpha, 1)}(x)=E_{1}^{(\alpha)}(x)-3 E_{0}^{(\alpha)}(x)=x-\frac{\alpha}{2}-3, \\
\hat{l}_{1,0}^{(\alpha, 2)}(x)=E_{1}^{(\alpha)}(x)-\frac{11}{2} E_{0}^{(\alpha)}(x)=x-\frac{\alpha}{2}-\frac{11}{2}, \\
\hat{l}_{1, j}^{(\alpha, 1)}(x)=\hat{l}_{1, j}^{(\alpha, 2)}(x)=0, \quad j \geq 2, \\
\hat{l}_{i, 0}^{(\alpha, 1)}(x)=E_{i}^{(\alpha)}(x)-3 E_{i-1}^{(\alpha)}(x)+\frac{5}{4} \sum_{k=0}^{i-2}(-2)^{i-k} E_{k}^{(\alpha)}(x), \\
\hat{l}_{i, 0}^{(\alpha, 2)}(x)=E_{i}^{(\alpha)}(x)-3 i E_{i-1}^{(\alpha)}(x)+\frac{5}{4} \sum_{k=1}^{i}(-2)^{k}\left(\begin{array}{l}
i \\
k
\end{array}\right) E_{i-k}^{(\alpha)}(x),
\end{gathered}
$$

for $i \geq 2$, and

$$
\begin{gathered}
\hat{l}_{i, 1}^{(\alpha, 1)}(x)=i E_{i-1}^{(\alpha)}(x)-3(i-1) E_{i-2}^{(\alpha)}(x) \\
+\frac{5}{4} \sum_{k=1}^{i-2}(-2)^{i-k} k E_{k-1}^{(\alpha)}(x),
\end{gathered}
$$

for $i \geq 3$.

The following results show some factorizations of $\mathscr{E}^{(\alpha)}(x)$ in terms of Fibonacci and Lucas matrices, respectively.

Theorem 5.The generalized Euler polynomial matrix $\mathscr{E}^{(\alpha)}(x)$ can be factorized in terms of the Fibonacci matrix $\mathscr{F}$, as follows:

$$
\mathscr{E}^{(\alpha)}(x)=\mathscr{F} \mathscr{M}^{(\alpha)}(x),
$$

or,

$$
\mathscr{E}^{(\alpha)}(x)=\mathscr{N}^{(\alpha)}(x) \mathscr{F}
$$

In particular,

$$
\begin{gathered}
\mathscr{F} \mathscr{M}(x)=\mathscr{E}(x)=\mathscr{N}(x) \mathscr{F}, \\
\mathscr{F} \mathscr{M}=\mathscr{E}=\mathscr{N} \mathscr{F},
\end{gathered}
$$

and

$$
\mathscr{F} \mathscr{M}\left(\frac{1}{2}\right)=\mathbb{E}=\mathscr{N}\left(\frac{1}{2}\right) \mathscr{F}
$$

Proof.Since the relation (27) is equivalent to $\mathscr{F}^{-1} \mathscr{E}(\alpha)(x)=\mathscr{M}^{(\alpha)}(x)$, it is possible to follow the proof given in [32, Theorem 4.1], making the corresponding modifications, for obtaining (27). The relation (28) can be obtained using a similar procedure. The relations (29), (30) and (31) are straightforward consequences of (27) and (28).

Also, the relations (27) and (28) allow us to deduce the following identity:

$$
\mathscr{M}^{(\alpha)}(x)=\mathscr{F}^{-1} \mathscr{N}^{(\alpha)}(x) \mathscr{F} .
$$

As a consequence of Theorems 4 and 5, we can derive simple factorizations for the inverses of the polynomial matrices $\mathscr{M}\left(x+\frac{1}{2}\right)$ and $\mathscr{N}\left(x+\frac{1}{2}\right)$ :

Corollary 5.The inverses of the polynomial matrices $\mathscr{M}\left(x+\frac{1}{2}\right)$ and $\mathscr{N}\left(x+\frac{1}{2}\right)$ can be factorized, as follows:

$$
\begin{aligned}
& {\left[\mathscr{M}\left(x+\frac{1}{2}\right)\right]^{-1}=\mathscr{D} P[-x] \mathscr{F},} \\
& {\left[\mathscr{N}\left(x+\frac{1}{2}\right)\right]^{-1}=\mathscr{F} \mathscr{D} P[-x] .}
\end{aligned}
$$


In particular,

$$
\begin{gathered}
\mathscr{M}^{-1}=\mathscr{D} P\left[\frac{1}{2}\right] \mathscr{F}, \quad \text { and } \quad \mathscr{N}^{-1}=\mathscr{F} \mathscr{D} P\left[\frac{1}{2}\right], \\
{\left[\mathscr{M}\left(\frac{1}{2}\right)\right]^{-1}=\mathscr{D} \mathscr{F}, \quad \text { and } \quad\left[\mathscr{N}\left(\frac{1}{2}\right)\right]^{-1}=\mathscr{F} \mathscr{D} .}
\end{gathered}
$$

An analogous reasoning as used in the proof of Theorem 5 allows us to prove the results below.

Theorem 6.The generalized Euler polynomial matrix $\mathscr{E}^{(\alpha)}(x)$ can be factorized in terms of the Lucas matrix $\mathscr{L}$, as follows:

$$
\mathscr{E}^{(\alpha)}(x)=\mathscr{L}_{1}^{(\alpha)}(x)
$$

or,

$$
\mathscr{E}^{(\alpha)}(x)=\mathscr{L}_{2}^{(\alpha)}(x) \mathscr{L}
$$

In particular,

$$
\begin{gathered}
\mathscr{L} \mathscr{L}_{1}(x)=\mathscr{E}(x)=\mathscr{L}_{2}(x) \mathscr{L}, \\
\mathscr{L} \mathscr{L}_{1}=\mathscr{E}=\mathscr{L}_{2} \mathscr{L}
\end{gathered}
$$

and

$$
\mathscr{L} \mathscr{L}_{1}^{\left(\frac{1}{2}\right)}(x)=\mathbb{E}=\mathscr{L}_{2}^{\left(\frac{1}{2}\right)}(x) \mathscr{L} .
$$

Also, the relations (32) and (33) allow us to deduce the following identity:

$$
\mathscr{L}_{1}^{(\alpha)}(x)=\mathscr{L}^{-1} \mathscr{L}_{2}^{(\alpha)}(x) \mathscr{L}
$$

Corollary 6.The inverses of the polynomial matrices $\mathscr{L}_{1}\left(x+\frac{1}{2}\right)$ and $\mathscr{L}_{2}\left(x+\frac{1}{2}\right)$ can be factorized, as follows:

$$
\begin{aligned}
& {\left[\mathscr{L}_{1}\left(x+\frac{1}{2}\right)\right]^{-1}=\mathscr{D} P[-x] \mathscr{L},} \\
& {\left[\mathscr{L}_{2}\left(x+\frac{1}{2}\right)\right]^{-1}=\mathscr{L} \mathscr{D} P[-x] .}
\end{aligned}
$$

In particular,

$$
\begin{gathered}
\mathscr{L}_{1}^{-1}=\mathscr{D} P\left[\frac{1}{2}\right] \mathscr{L}, \quad \text { and } \quad \mathscr{L}_{2}^{-1}=\mathscr{L} \mathscr{D} P\left[\frac{1}{2}\right], \\
{\left[\mathscr{L}_{1}\left(\frac{1}{2}\right)\right]^{-1}=\mathscr{D} \mathscr{L}, \quad \text { and } \quad\left[\mathscr{L}_{2}\left(\frac{1}{2}\right)\right]^{-1}=\mathscr{L} \mathscr{D} .}
\end{gathered}
$$

Remark.If we consider $a \in \mathbb{C}, b \in \mathbb{C} \backslash\{0\}$ and $s=0,1$, then Theorems 5 and 6 , as well as their corollaries have corresponding analogous forms for generalized Fibonacci matrices of type $s, \mathscr{F}^{(a, b, s)}$, and generalized Fibonacci matrices $\mathscr{U}^{(a, b, 0)}$ with second order recurrent sequence $U_{n}^{(a, b)}$ subordinated to certain constraints [28].
Another identities involving Fibonacci and Lucas numbers as well as the generalized Euler polynomials and numbers are, as follows:

Theorem 7.For $0 \leq r \leq n$ and $\alpha$ any real or complex number, we have

$$
\begin{aligned}
\left(\begin{array}{c}
n \\
r
\end{array}\right) E_{n-r}^{(\alpha)}(x)= & F_{n-r+1}+\left[(r+1) x-\frac{(r+1) \alpha+2}{2}\right] F_{n-r} \\
& +\sum_{k=r+2}^{n}\left(\begin{array}{c}
k \\
r
\end{array}\right)\left\{E_{k-r}^{(\alpha)}(x)-\frac{k-r}{k}\left[E_{k-r-1}^{(\alpha)}(x)\right.\right. \\
& \left.\left.+\frac{k-r-1}{k-1} E_{k-r-2}^{(\alpha)}(x)\right]\right\} F_{n-k+1} \\
= & F_{n-r+1}+\left[n\left(x-\frac{\alpha}{2}\right)-1\right] F_{n-r} \\
& +\sum_{k=0}^{n-2}\left(\begin{array}{l}
n \\
k
\end{array}\right)\left\{E_{n-k}^{(\alpha)}(x)-\frac{n-k}{k+1}\left[E_{n-k-1}^{(\alpha)}(x)\right.\right. \\
& \left.\left.+\frac{n-k-1}{k+2} E_{n-k-2}^{(\alpha)}(x)\right]\right\} F_{k-r+1} .
\end{aligned}
$$

Proof.We proceed as in the proof of [32, Theorem 4.2], making the corresponding modifications. From (26), it is clear that

$$
\tilde{m}_{r, r}^{(\alpha)}(x)=1, \quad \tilde{m}_{r+1, r}^{(\alpha)}(x)=(r+1) x-\frac{(r+1) \alpha+2}{2},
$$

and, for $k \geq r+2$ :

$$
\begin{aligned}
\tilde{m}_{k, r}^{(\alpha)}(x)= & \left(\begin{array}{l}
k \\
r
\end{array}\right)\left\{E_{k-r}^{(\alpha)}(x)-\frac{k-r}{k}\left[E_{k-r-1}^{(\alpha)}(x)\right.\right. \\
& \left.\left.+\frac{k-r-1}{k-1} E_{k-r-2}^{(\alpha)}(x)\right]\right\} .
\end{aligned}
$$

Next, it follows from (27) that

$$
\begin{aligned}
\left(\begin{array}{l}
n \\
r
\end{array}\right) E_{n-r}^{(\alpha)}(x)= & E_{n, r}^{(\alpha)}(x)=\sum_{k=r}^{n} F_{n-k+1} \tilde{m}_{k, r}^{(\alpha)}(x) \\
= & F_{n-r+1}+F_{n-r} \tilde{m}_{r+1, r}^{(\alpha)}(x) \\
& +\sum_{k=r+2}^{n} F_{n-k+1} \tilde{m}_{k, r}^{(\alpha)}(x),
\end{aligned}
$$

so, 


$$
\begin{aligned}
\left(\begin{array}{l}
n \\
r
\end{array}\right) E_{n-r}^{(\alpha)}(x)= & F_{n-r+1}+\left[(r+1) x-\frac{(r+1) \alpha+2}{2}\right] F_{n-r} \\
& +\sum_{k=r+2}^{n}\left(\begin{array}{c}
k \\
r
\end{array}\right)\left\{E_{k-r}^{(\alpha)}(x)-\frac{k-r}{k}\left[E_{k-r-1}^{(\alpha)}(x)\right.\right. \\
& \left.\left.+\frac{k-r-1}{k-1} E_{k-r-2}^{(\alpha)}(x)\right]\right\} F_{n-k+1} .
\end{aligned}
$$

This chain of equalities completes the first part of the proof. The second one is obtained in a similar way, taking into account the following identities:

$$
\tilde{n}_{n, n}^{(\alpha)}(x)=1, \quad \tilde{n}_{n, n-1}^{(\alpha)}(x)=n\left(x-\frac{\alpha}{2}\right)-1,
$$

and, for $0 \leq k \leq n-2$ :

$$
\begin{aligned}
\tilde{n}_{n, k}^{(\alpha)}(x)= & \left(\begin{array}{l}
n \\
k
\end{array}\right)\left\{E_{n-k}^{(\alpha)}(x)-\frac{n-k}{k+1}\left[E_{n-k-1}^{(\alpha)}(x)\right.\right. \\
& \left.\left.+\frac{n-k-1}{k+2} E_{n-k-2}^{(\alpha)}(x)\right]\right\} .
\end{aligned}
$$

Corollary 7.For $0 \leq r \leq n$ and $\alpha$ any real number, we have

$$
\begin{aligned}
& (-1)^{n}\left(\begin{array}{l}
n \\
r
\end{array}\right) E_{n-r}^{(\alpha)}(x)=(-1)^{r} F_{n-r+1} \\
+ & (-1)^{r+1}\left[\frac{(r+1)(2 x-\alpha)+2}{2}\right] F_{n-r} \\
& +\sum_{k=r+2}^{n}(-1)^{k}\left(\begin{array}{l}
k \\
r
\end{array}\right)\left\{E_{k-r}^{(\alpha)}(x)+\frac{k-r}{k}\left[E_{k-r-1}^{(\alpha)}(x)\right.\right. \\
- & \left.\left.\frac{k-r-1}{k-1} E_{k-r-2}^{(\alpha)}(x)\right]\right\} F_{n-k+1} \\
= & (-1)^{r} F_{n-r+1}+(-1)^{r+1}\left[n\left(x-\frac{\alpha}{2}\right)-1\right] F_{n-r} \\
+ & \sum_{k=0}^{n-2}(-1)^{n-k+r}\left(\begin{array}{l}
n \\
k
\end{array}\right)\left\{E_{n-k}^{(\alpha)}(x)+\frac{n-k}{k+1}\left[E_{n-k-1}^{(\alpha)}(x)\right.\right. \\
+ & \left.\left.\frac{n-k-1}{k+2} E_{n-k-2}^{(\alpha)}(x)\right]\right\} F_{k-r+1} .
\end{aligned}
$$

Proof.Replacing $x$ by $\alpha-x$ in (34) and applying the formula

$$
E_{n}^{(\alpha)}(x)=(-1)^{n} E_{n}^{(\alpha)}(\alpha-x)
$$

to the resulting identity, we obtain the first identity of Corollary 7. An analogous reasoning yields the second identity.

Analogous reasonings to those used in the proofs of Theorem 7 and Corollary 7 allow us to prove the following results.
Theorem 8.For any real or complex number $\alpha$, we have the following identities

$$
\begin{aligned}
E_{n}^{(\alpha)}(x)= & L_{n+1}+\left(x-\frac{\alpha}{2}-3\right) L_{n} \\
& +\sum_{k=2}^{n}\left(E_{k}^{(\alpha)}(x)-3 E_{k-1}^{(\alpha)}(x)\right) L_{n-k+1} \\
& +5 \sum_{k=2}^{n} \sum_{s=0}^{k-2}(-1)^{k-s} 2^{k-s-2} L_{n-k+1} E_{s}^{(\alpha)}(x),
\end{aligned}
$$

whenever $n \geq 2$.

$$
\begin{aligned}
n E_{n-1}^{(\alpha)}(x)= & L_{n}+(2 x-\alpha-3) L_{n-1} \\
& +\sum_{k=3}^{n}\left(k E_{k-1}^{(\alpha)}(x)-3(k-1) E_{k-2}^{(\alpha)}(x)\right) L_{n-k+1} \\
& +5 \sum_{k=3}^{n} \sum_{s=1}^{k-2}(-1)^{k-s} 2^{k-s-2} s L_{n-k+1} E_{s-1}^{(\alpha)}(x),
\end{aligned}
$$

whenever $n \geq 3$.

\section{Corollary 8.The following identities hold.}

$$
\begin{aligned}
(-1)^{n} E_{n}^{(\alpha)}(x)= & L_{n+1}-\left(x-\frac{\alpha}{2}+3\right) L_{n} \\
& +\sum_{k=2}^{n}(-1)^{k}\left(E_{k}^{(\alpha)}(x)+3 E_{k-1}^{(\alpha)}(x)\right) L_{n-k+1} \\
& +5 \sum_{k=2}^{n} \sum_{s=0}^{k-2}(-1)^{k-s} 2^{k-s-2} L_{n-k+1} E_{s}^{(\alpha)}(x),
\end{aligned}
$$

whenever $n \geq 2$.

$$
\begin{aligned}
(-1)^{n-1} n E_{n-1}^{(\alpha)}(x)= & L_{n}+(\alpha-2 x-3) L_{n-1} \\
& +\sum_{k=3}^{n}(-1)^{k-1}\left(k E_{k-1}^{(\alpha)}(x)\right. \\
& \left.+3(k-1) E_{k-2}^{(\alpha)}(x)\right) L_{n-k+1} \\
& +5 \sum_{k=3}^{n} \sum_{s=1}^{k-2}(-1)^{k-1} 2^{k-s-2} s L_{n-k+1} E_{s-1}^{(\alpha)}(x),
\end{aligned}
$$

whenever $n \geq 3$.

By (35), (36), (37) and (38), we obtain the following interesting identities involving Lucas and Euler numbers.

-For $n \geq 2$ :

$$
\begin{aligned}
E_{n}-\left(L_{n+1}-\frac{7}{2} L_{n}\right) & =\sum_{k=2}^{n}\left(E_{k}-3 E_{k-1}\right. \\
& \left.=+5 \sum_{s=0}^{k-2}(-1)^{k-s} 2^{k-s-2} E_{s}\right) L_{n-k+1},
\end{aligned}
$$




$$
\begin{aligned}
(-1)^{n} E_{n}= & L_{n+1}-\frac{5}{2} L_{n} \\
& +\sum_{k=2}^{n}(-1)^{k}\left(E_{k}+3 E_{k-1}\right) L_{n-k+1} \\
& +5 \sum_{k=2}^{n} \sum_{s=0}^{k-2}(-1)^{k-s} 2^{k-s-2} L_{n-k+1} E_{s} .
\end{aligned}
$$

-For $n \geq 3$ :

$$
\begin{gathered}
n E_{n-1}-\left(L_{n}-4 L_{n-1}\right)= \\
\sum_{k=3}^{n}\left(k E_{k-1}-3(k-1) E_{k-2}\right) L_{n-k+1} \\
+5 \sum_{k=3}^{n} \sum_{s=1}^{k-2}(-1)^{k-s} 2^{k-s-2} s E_{s-1} L_{n-k+1}, \\
(-1)^{n-1} n E_{n-1}-L_{n}-(\alpha-2 x-3) L_{n-1}= \\
\sum_{k=3}^{n}(-1)^{k-1}\left(k E_{k-1}+3(k-1) E_{k-2}\right) L_{n-k+1} \\
+5 \sum_{k=3}^{n} \sum_{s=1}^{k-2}(-1)^{k-1} 2^{k-s-2} s L_{n-k+1} E_{s-1} .
\end{gathered}
$$

Other similar combinatorial identities may be obtained using the results of [14]. We leave their formulation to the interested reader.

\section{Euler matrices and their relation with Stirling and Vandermonde matrices}

Let $s(n, k)$ and $S(n, k)$ be the Stirling numbers of the first and second types, which are respectively defined by the generating functions [21, Chapther 1, Section 1.6]:

$$
\begin{aligned}
\sum_{k=0}^{n} s(n, k) z^{k} & =z(z-1) \cdots(z-n+1), \\
(\log (1+z))^{k} & =k ! \sum_{n=k}^{\infty} s(n, k) \frac{z^{n}}{n !}, \quad|z|<1, \\
z^{n} & =\sum_{k=0}^{n} S(n, k) z(z-1) \cdots(z-k+1), \\
\left(e^{z}-1\right)^{k} & =k ! \sum_{n=k}^{\infty} S(n, k) \frac{z^{n}}{n !} .
\end{aligned}
$$

The value $|s(n, k)|$ represents the number of permutations of $n$ elements with $k$ disjoint cycles. While, the Stirling numbers of the second type $S(n, k)$ give the number of partitions of $n$ objects into $k$ non-empty subsets. Another way to compute these numbers is by means of the formula (see [6, Eq. (5.1)] or [19, p. 226]):

$$
S(n, k)=\frac{1}{k !} \sum_{l=0}^{k}(-1)^{k-l}\left(\begin{array}{l}
k \\
l
\end{array}\right) l^{n}, \quad 1 \leq k \leq n .
$$

A recent connection between the Stirling numbers of the second type and the Euler polynomials is given by the formula (see [7, Theorem 3.1, Eq. (3.3)]):

$$
\begin{aligned}
E_{n}(x)= & \sum_{k=0}^{n}(-1)^{n-k}\left(\begin{array}{l}
n \\
k
\end{array}\right)\left[\sum_{l=1}^{n-k+1} \frac{(-1)^{l-1}(l-1) !}{2^{l-1}}\right. \\
& \times S(n-k+1, l)] x^{k} .
\end{aligned}
$$

Proceeding as in the proof of [7, Theorem 3.1], one can find a similar relation to the previous one, but connecting Stirling numbers of the first type and a particular class of generalized Euler polynomials.

Theorem 9.Let us assume that $\alpha=m \in \mathbb{N}$. Then, the connection between the Stirling numbers of the first kind and the generalized Euler polynomial $E_{n}^{(m)}(x)$ is given by the formula:

$$
E_{n}^{(m)}(x)=\frac{1}{2^{n}} \sum_{k=0}^{n}\left(\begin{array}{l}
n \\
k
\end{array}\right)\left[\sum_{j=0}^{n-k} s(n-k, j)(-m)^{j}\right](2 x)^{k}
$$

Proof.By Leibniz's theorem for differentiation, we have

$$
\begin{aligned}
& \frac{\partial^{r}}{\partial z^{r}}\left[\left(\frac{2}{e^{z}+1}\right)^{m} e^{x z}\right] \\
= & \sum_{k=0}^{r}\left(\begin{array}{l}
r \\
k
\end{array}\right)\left[\left(\frac{2}{e^{z}+1}\right)^{m}\right]^{(k)} \frac{\partial^{r-k}}{\partial z^{r-k}}\left(e^{x z}\right) \\
= & \sum_{k=0}^{r}\left(\begin{array}{l}
r \\
k
\end{array}\right)\left[\left(\frac{2}{e^{z}+1}\right)^{m}\right]^{(k)} x^{r-k} e^{x z} \\
= & \left(\frac{2}{e^{z}+1}\right)^{m} e^{(x+1) z} \sum_{k=0}^{r}\left(\begin{array}{l}
r \\
k
\end{array}\right) \frac{(-m)_{k} x^{r-k}}{\left(e^{z}+1\right)^{k}},
\end{aligned}
$$

where in the last expression, $(-m)_{k}$ denotes the falling factorial with opposite argument $-m$.

Combining this with the $r$-th differentiation on both sides of the generating function in (1) reveals that

$$
\begin{aligned}
& \sum_{n=r}^{\infty} E_{n}^{(m)}(x) \frac{z^{n-r}}{(n-r) !}= \\
&\left(\frac{2}{e^{z}+1}\right)^{m} e^{(x+1) z} \sum_{k=0}^{r}\left(\begin{array}{l}
r \\
k
\end{array}\right) \frac{(-m)_{k} x^{r-k}}{\left(e^{z}+1\right)^{k}} .
\end{aligned}
$$

Further taking $z \rightarrow 0$ and employing (39) give

$$
\begin{aligned}
E_{r}^{(m)}(x) & =\sum_{k=0}^{r}\left(\begin{array}{l}
r \\
k
\end{array}\right)(-m)_{k} \frac{x^{r-k}}{2^{k}} \\
& =\frac{1}{2^{r}} \sum_{k=0}^{r}\left(\begin{array}{l}
r \\
k
\end{array}\right)(-m)_{r-k}(2 x)^{k} \\
& =\frac{1}{2^{r}} \sum_{k=0}^{r}\left(\begin{array}{l}
r \\
k
\end{array}\right)\left[\sum_{j=0}^{r-k} s(r-k, j)(-m)^{j}\right](2 x)^{k} .
\end{aligned}
$$


Finally, changing $r$ by $n$, the proof of the formula (41) is completed.

Definition 5.For the Stirling numbers $s(i, j)$ and $S(i, j)$ of the first and the second types, respectively, define $\mathfrak{S}$ and $\mathscr{S}$ to be the $(n+1) \times(n+1)$ matrices by

$$
\begin{gathered}
\mathfrak{S}_{i, j}=\{s(i, j), \quad i \geq j, 0, \quad \text { otherwise, and } \\
\mathscr{S}_{i, j}=\left\{\begin{array}{l}
S(i, j), \quad i \geq j, \\
0, \quad \text { otherwise. }
\end{array}\right.
\end{gathered}
$$

The matrices $\mathfrak{S}$ and $\mathscr{S}$ are called Stirling matrix of the first and the second types, respectively (see [3]).

To obtain factorizations for Euler matrices via Stirling matrices of the first type, we will need the following matrix: For $m \in \mathbb{N}$, let $\mathfrak{S}^{(m)}$ be the $(n+1) \times(n+1)$ matrix whose $(i, j)$-entries are defined by

$$
\mathfrak{S}_{i, j}^{(m)}=\left\{\begin{array}{l}
\left(\begin{array}{c}
i \\
j
\end{array}\right) \sum_{k=0}^{i-j} s(i-j, k)(-m)^{k}, \quad i \geq j, \\
0, \quad \text { otherwise. }
\end{array}\right.
$$

Next theorem shows the corresponding factorizations of the generalized Euler matrix $\mathscr{E}^{(m)}, m \in \mathbb{N}$, in terms of Stirling matrices of the first type, when the expression (41) is incorporated.

Theorem 10.For $m \in \mathbb{N}$, the generalized Euler matrix $\mathscr{E}^{(m)}(x)$ can be factorized, as follows:

$$
\mathscr{E}^{(m)}(x)=\mathfrak{S}^{(m)} P[x] .
$$

Proof.For $m \in \mathbb{N}$ and $i \geq j$, let $A_{i, j}^{(m)}(x)$ be the $(i, j)$-th entry of the matrix product $\mathfrak{S}^{(m)} P[x]$, then

$$
\begin{aligned}
A_{i, j}^{(m)}(x) & =\sum_{k=j}^{i} \mathfrak{S}_{i, k}^{(m)} p_{k, j}(x) \\
& =\sum_{k=j}^{i}\left(\begin{array}{l}
i \\
k
\end{array}\right)\left(\begin{array}{l}
k \\
j
\end{array}\right)\left[\sum_{r=0}^{i-k} s(i-k, r)(-m)^{r}\right] x^{k-j} \\
& =\sum_{k=j}^{i}\left(\begin{array}{l}
i \\
j
\end{array}\right)\left(\begin{array}{c}
i-j \\
k-j
\end{array}\right) 2^{j-i}\left[\sum_{r=0}^{i-k} s(i-k, r)(-m)^{r}\right](2 x)^{k-j} \\
& =\frac{\left(\begin{array}{l}
i \\
j
\end{array}\right)}{2^{i-j}} \sum_{k=0}^{i-j}\left(\begin{array}{c}
i-j \\
k
\end{array}\right)\left[\sum_{r=0}^{i-j-k} s(i-j-k, r)(-m)^{r}\right](2 x)^{k} \\
& =\left(\begin{array}{c}
i \\
j
\end{array}\right) E_{i-j}^{(m)}(x),
\end{aligned}
$$

The last equality is an immediate consequence of (41), and (42) follows from the previous chain of equalities.
Remark.Notice that using (40) and putting $m=1$ into (42), we get

$$
\begin{aligned}
\left(\begin{array}{c}
i \\
j
\end{array}\right) E_{i-j}(x)= & A_{i, j}^{(1)}(x) \\
= & (-1)^{i-j}\left(\begin{array}{l}
i \\
j
\end{array}\right) \sum_{k=0}^{i-j}(-1)^{k}\left(\begin{array}{c}
i-j \\
k
\end{array}\right) \\
& \times\left[\sum_{r=0}^{i-j-k}\left(-\frac{1}{2}\right)^{r} r ! S(i-j-k+1, r+1)\right] x^{k} .
\end{aligned}
$$

Definition 6.The $(n+1) \times(n+1)$ shifted Euler polynomial matrix $\tilde{\mathscr{E}}(x)$ is given by

$$
\tilde{\mathscr{E}}_{i, j}(x)=\mathscr{E}_{i}(j+x), \quad 0 \leq i, j \leq n .
$$

Let us consider the Vandermonde matrix:

$$
\mathscr{V}(x):=\left[\begin{array}{ccccc}
1 & 1 & 1 & \cdots & 1 \\
x & 1+x & 2+x & \cdots & n+x \\
x^{2} & (1+x)^{2} & (2+x)^{2} & \cdots & (n+x)^{2} \\
\vdots & \vdots & \vdots & \ddots & \vdots \\
x^{n} & (1+x)^{n} & (2+x)^{n} & \cdots & (n+x)^{n}
\end{array}\right] .
$$

In [4, Theorem 2.1], the following factorization for the Vandermonde matrix $\mathscr{V}(x)$ was stated.

$$
\begin{aligned}
\mathscr{V}(x) & =\left([1] \oplus \tilde{S}_{n+1}\right) \Delta_{n+1}(x) P^{T} \\
& :=\left([1] \oplus \tilde{S}_{n+1}\right) \Delta_{n+1}(x)(P[1])^{T},
\end{aligned}
$$

where $\tilde{S}_{n+1}$ is the factorial Stirling matrix, i.e. the $(n+1) \times(n+1)$ matrix whose $(i, j)$-th entry is given by $\tilde{S}_{i, j, n+1}:=j ! \mathscr{S}_{i, j}, \quad i \geq j$ and otherwise $0, \quad$ and $\Delta_{n+1}(x)(P[1])^{T}$ represents the LU-factorization of a lower triangular matrix whose $(i, j)$-th entry is $\left(\begin{array}{c}x \\ i-j\end{array}\right)$, if $i \geq j$ and otherwise 0 .

The relation between the shifted Euler polynomial matrix $\tilde{\mathscr{E}}(x)$ and the matrices $\mathscr{V}(x)$ and $\tilde{S}_{n+1}$ is contained in the following result.

Theorem 11.The shifted Euler polynomial matrix $\tilde{\mathscr{E}}(x)$ can be factorized in terms of the Vandermonde matrix $\mathscr{V}(x)$, and thereby in terms of the factorial Stirling matrix $\tilde{S}_{n+1}$, as follows:

$$
\begin{gathered}
\tilde{\mathscr{E}}(x)=\mathscr{E} \mathscr{V}(x), \\
\tilde{\mathscr{E}}(x)=\mathscr{E}\left([1] \oplus \tilde{S}_{n+1}\right) \Delta_{n+1}(x) P^{T} .
\end{gathered}
$$

Proof.Let $\tilde{\mathscr{E}}_{i, j}(x)$ be the $(i, j)$-th entry of the shifted Euler polynomial matrix $\tilde{\mathscr{E}}(x)$. Then, using (9), we get

$\tilde{\mathscr{E}}_{i, j}(x)=\mathscr{E}_{i}(j+x)=\sum_{k=0}^{i}\left(\begin{array}{l}i \\ k\end{array}\right) E_{i-k}(j+x)^{k}=\sum_{k=0}^{i} E_{i, k} \mathscr{N}_{k, j}(x)$.

Hence, (44) follows from this chain of equalities. The relation (45) is a straightforward consequence of (43). 
Remark. Note that the relations (44) and (45) are the analogous of [32, Eqs. (37), (38)], respectively, in the context of Euler polynomial matrices.

In the present paper, all matrix identities have been expressed using finite matrices. Since such matrix identities involve lower triangular matrices, they have a resemblance for infinite matrices. For instance, let $\mathscr{E}_{\infty}\left(x+\frac{1}{2}\right), P_{\infty}[x]$ and $\mathbb{E}_{\infty}$ be the infinite cases of the matrices $\mathscr{E}\left(x+\frac{1}{2}\right), P[x]$ and $\mathbb{E}$, respectively. Then, the following identity holds

$$
\mathscr{E}_{\infty}\left(x+\frac{1}{2}\right)=P_{\infty}[x] \mathbb{E}_{\infty}
$$

We leave the formulation of the other analogous identities to the reader.

Finally, in addition to Theorem 11, we would like to state that an interesting application in connection with the subjects treated in the present paper is its use in the LU-factorization of certain class of matrices, whose components can be represented by a Wronskian of some function set (cf., for instance [30] for the case of generalized Pascal functional matrices).

\section{Perspective}

We have showed some new algebraic properties of the generalized Euler polynomial matrix $\mathscr{E}^{(\alpha)}(x)$ and the Euler matrix $\mathscr{E}$. Taking into account some properties of Euler polynomials and numbers, we have deduced product formulae for $\mathscr{E}^{(\alpha)}(x)$ and defined the inverse matrix of $\mathscr{E}$. Also, we have established some explicit expressions for the Euler polynomial matrix $\mathscr{E}(x)$, which involves the generalized Pascal, Fibonacci and Lucas matrices, respectively.

The paper [11] addressed an explicit formula to the inverse matrix of the $q$-Pascal matrix plus one in terms of the $q$-analogue of the Euler matrix $\mathscr{E}$. Thus, combining some results of the present paper helps define the inverse matrix of the $q$-analogue of the specialized Euler matrix $\mathbb{E}$. This inverse matrix allows us to establish the analogous of Theorem 3 in the framework of the class of generalized $q$-Pascal matrices.

A future work has to address the exploration of similar matrix identities in the setting of the $q$-umbral calculus. The notion of commutativity with respect to the parameter $q$ appears when $\mathbb{R}$ is endowed with some structure of alphabet and its elements are called letters or umbrae (see e.g. [6, Section 4.1], for the details). If $\mathbb{R}$ is regarded as a set generated by itself together with the operations of NWA $q$-addition, NWA $q$-subtraction, JHC $q$-addition and JHC $q$-subtraction, then the algebraic structure of $\mathbb{R}$ as such an alphabet is different from the usual algebraic structure of $\mathbb{R}$ as real number field. Moreover, $\mathbb{R}$ has structure of commutative semigroup with each one of these $q$-operations (see [6, Definition 25]).

For instance, the version of the generalized Pascal matrix of first type in this context was introduced in [34], as follows: Let $x$ be any nonzero real number. The $q$-Pascal matrix $\mathscr{P}[x]$ is an $(n+1) \times(n+1)$ matrix whose $(i, j)$-entries are given by:

$$
p_{i, j, q}(x)=\left\{\begin{array}{l}
{\left[\begin{array}{l}
i \\
j
\end{array}\right]_{q} x^{i-j}, \quad i \geq j,} \\
0, \quad \text { otherwise }
\end{array}\right.
$$

where $\left[\begin{array}{l}i \\ j\end{array}\right]_{q}$ denotes the $q$-binomial coefficient.

In $[11,34]$, some properties of the $q$-Pascal matrix are shown, for example, its matrix factorization by special summation matrices, the factorization of its inverse matrix, and its relation with the $q$-analogue of the generalized Bernoulli matrix.

The $q$-Euler polynomials $E_{n, q}(x)$ and the generalized $q$-Euler polynomials $E_{n, q}^{(\alpha)}(x)$ of (real or complex) order $\alpha$, are defined, as follows (see $[11,12])$ :

$$
\left(\frac{2}{e_{q}(z)+1}\right)^{\alpha} e_{q}(x z)=\sum_{n=0}^{\infty} E_{n, q}^{(\alpha)}(x) \frac{z^{n}}{[n]_{q} !},
$$

where $|z|<\pi, 1^{\alpha}:=1$, and

$$
E_{n, q}(x):=E_{n}^{(1)}(x), \quad n \in \mathbb{N}_{0} .
$$

As usual, the numbers $E_{n, q}^{(\alpha)}:=E_{n, q}^{(\alpha)}(0)$ and $E_{n, q}:=E_{n, q}(0)$ are called the generalized $q$-Euler numbers of order $\alpha$ and the generalized $q$-Euler numbers, respectively. We introduce the $q$-analogue of the classical Euler numbers by means of the following generating function

$$
\frac{2}{e_{q}(z)+e_{q}(-z)}=\sum_{n=0}^{\infty} \varepsilon_{n, q} \frac{z^{n}}{[n]_{q} !} .
$$

From (47) and (48), it is possible to check that if 1 commutes with -1 , the connection between the $q$-analogue of the classical Euler numbers and the $q$-Euler polynomials is given by the formula

$$
\varepsilon_{n, q}=2^{n} E_{n, q}\left(\frac{1}{2}\right), \quad n \in \mathbb{N}_{0} .
$$

Finally:

(a)The $q$-Euler polynomials $E_{n, q}(x)$ are different from those defined by Srivastava and Choi in [21, Section 6.7].

(b)For $\alpha=m \in \mathbb{N}$, the generalized $q$-Euler polynomials $E_{n, q}^{(\alpha)}(x) \quad$ coincide with the so-called Nalli-Ward-Al-Salam $q$-Euler polynomials (in short, NWA $q$-Euler polynomials), $F_{\mathrm{NWA}, n, q}^{(m)}(x)$ (see for instance, [6, Eq. (4.196)]). 
(c)From the generating relation (46), it is fairly straightforward to deduce the addition formula:

$$
E_{n, q}^{(\alpha+\beta)}(x+y)=\sum_{k=0}^{n}\left[\begin{array}{l}
n \\
k
\end{array}\right]_{q} E_{k, q}^{(\alpha)}(x) E_{n-k, q}^{(\beta)}(y),
$$

provided that $x$ commutes with $y$ (cf., [6, Eq. (4.7)]).

(d)Making the substitution $\beta=0$ into (49) and interchanging $x$ and $y$, we get

$$
E_{n, q}^{(\alpha)}(x+y)=\sum_{k=0}^{n}\left[\begin{array}{l}
n \\
k
\end{array}\right]_{q} E_{k, q}^{(\alpha)}(y) x^{n-k} .
$$

\section{Acknowledgement}

The first author (YQ) has been supported by the grants Impacto Caribe (IC-002627-2015) from Universidad del Atlántico, Colombia, and DID-USB (S1-IC-CB-003-16) from Decanato de Investigación y Desarrollo. Universidad Simón Bolívar, Venezuela.

The second (WR) and third (AU) authors have been supported by the grant Impacto Caribe (IC-002627-2015) from Universidad del Atlántico, Colombia.

The authors are so grateful to the anonymous reviewers for their careful checking of the details and the helpful comments and suggestions that improved this paper.

\section{References}

[1] T. Arakawa, T. Ibukiyama and M. Kaneko, Bernoulli Numbers and Zeta Functions, Springer, London UK, 51-63, (2014).

[2] G. S. Call and D. J. Velleman, Pascal's matrices, Amer. Math. Monthly, 100, 372-376 (1993).

[3] G.-S. Cheon and J.-S. Kim, Stirling matrix via Pascal matrix, Linear Algebra Appl., 329, 49-59 (2001).

[4] G.-S. Cheon and J.-S. Kim, Factorial Stirling matrix and related combinatorial sequences, Linear Algebra Appl., 357, 247-258 (2002).

[5] L. Comtet, Advanced Combinatorics. The Art of Finite and Infinite Expansions, D. Reidel Publishing Co., Boston US, 204-219, (1974).

[6] T. Ernst, A Comprehensive Treatment of q-Calculus, Birkhäuser, London UK, 97-167, (2012).

[7] B.-N. Guo and F. Qi, Explicit formulae for computing Euler polynomials in terms of Stirling numbers of the second kind, J. Comput. Appl. Math., 272, 251-257 (2014).

[8] Y. He, S. Araci and H. M. Srivastava, Some new formulas for the products of the Apostol type polynomials, Adv. Differ. Equ., 2016, 1-18 (2016).

[9] Y. He, S. Araci, H. M. Srivastava and M. Acikgöz, Some new identities for the Apostol-Bernoulli polynomials and the Apostol-Genocchi polynomials. Appl. Math. Comput., 262, 31-41 (2015).

[10] P. Hernández-Llanos, Y. Quintana and A. Urieles, About extensions of generalized Apostol-type polynomials, Results Math., 68, 203-225 (2015).
[11] G. I. Infante, J. L. Ramírez and A. Şahin, Some results on $q$-analogue of the Bernoulli, Euler and Fibonacci matrices, Math. Rep. (Bucur.), 19(69), 399-417 (2017).

[12] D. S. Kim and T. Kim, Some identities of $q$-Euler polynomials arising from $q$-umbral calculus, J. Inequal. Appl., 2014:1, 1-12 (2014).

[13] G.-Y. Lee, J.-S. Kim and S.-G. Lee, Factorizations and eigenvalues of Fibonacci and symmetric Fibonacci matrices, Fibonacci Quart., 40, 203-211 (2002).

[14] G.-Y. Lee, J.-S. Kim and S.-H. Cho, Some combinatorial identities via Fibonacci numbers, Discrete Appl. Math., 130, 527-534 (2003).

[15] Q.-M. Luo and H. M. Srivastava, Some generalizations of the Apostol-Bernoulli and Apostol-Euler polynomials, $J$. Math. Anal. Appl., 308, 290-302 (2005).

[16] N. E. Nørlund, Vorlesungen über Differenzenrechnung, Springer-Verlag, Berlin DE, 120-162, (1924).

[17] Á. Pintér and H. M. Srivastava, Addition theorems for the Appell polynomials and the associated classes of polynomial expansions, Aequationes Math., 85, 483-495 (2013).

[18] Y. Quintana, W. Ramírez and A. Urieles, On an operational matrix method based on generalized Bernoulli polynomials of level $m$, Calcolo 55, 1-29 (2018).

[19] J. Riordan, Combinatorial Identities, John Wiley \& Sons, Inc., New York US, 99-127, (1968).

[20] H. M. Srivastava, M. A. Boutiche and M. Rahmani, A class of Frobenius-type Eulerian polynomials, Rocky Mountain J. Math., 48, 1003-1013 (2018).

[21] H. M. Srivastava and J. Choi, Zeta and q-Zeta Functions and Associated Series and Integrals, Elsevier, London UK, 76-140, (2012).

[22] H. M. Srivastava, I. Kucukoğlu and Y. Simsek, Partial differential equations for a new family of numbers and polynomials unifying the Apostol-type numbers and the Apostol-type polynomials, J. Number Theory, 181, 117-146 (2017).

[23] H. M. Srivastava and H. L. Manocha, A Treatise on Generating Functions, Ellis Horwood Ltd., West Sussex UK, 384-402, (1984).

[24] H. M. Srivastava, M. Masjed-Jamei and M. Reza Beyki, A parametric type of the Apostol-Bernoulli, Apostol-Euler and Apostol-Genocchi polynomials, Appl. Math. Inform. Sci., 12, 907-916 (2018).

[25] H. M. Srivastava, M. A. Özarslan and C. Kaanuglu, Some generalized Lagrange-based Apostol-Bernoulli, ApostolEuler and Apostol-Genocchi polynomials, Russian J. Math. Phys., 20, 110-120 (2013).

[26] H. M. Srivastava, M. A. Özarslan and B. Yilmaz, Some families of differential equations associated with the Hermitebased Appell polynomials and other classes of Hermite-based polynomials, Filomat, 28, 695-708 (2014).

[27] H. M. Srivastava and Á. Pintér, Remarks on some relationships between the Bernoulli and Euler polynomials, Appl. Math. Lett., 17, 375-380 (2004).

[28] P. Stanimirović, J. Nikolov and I. Stanimirović, A generalization of Fibonacci and Lucas matrices, Discrete Appl. Math., 156, 2606-2619 (2008).

[29] Z. Z. Zhang, The linear algebra of generalized Pascal matrix, Linear Algebra Appl., 250, 51-60 (1997).

[30] Y. Yang and C. Micek, Generalized Pascal function matrix and its applications, Linear Algebra Appl., 423, 230-245 (2007). 
[31] Z. Z. Zhang and M. X. Liu, An extension of generalized Pascal matrix and its algebraic properties, Linear Algebra Appl., 271, 169-177 (1998).

[32] Z. Zhang and J. Wang, Bernoulli matrix and its algebraic properties, Discrete Appl. Math., 154, 1622-1632 (2006).

[33] Z. Zhang and Y. Zhang, The Lucas matrix and some combinatorial identities, Indian J. Pure Appl. Math., 38, 457465 (2007)

[34] D. Zheng, $q$-analogue of the Pascal matrix, Ars Combin., 87, 321-336 (2008).

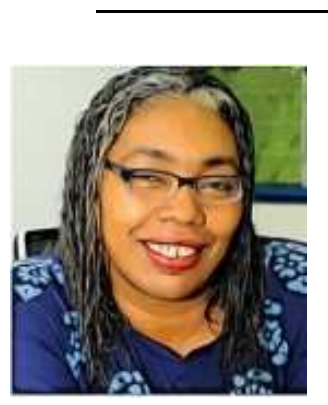

Yamilet Quintana

is currently Full Professor

in Department of Pure and Applied Mathematics at Universidad Simón Bolívar (Venezuela). She received her $\mathrm{PhD}$ in Mathematics from Universidad Central de Venezuela in 2004

Her research interest includes approximation theory and special functions, mathematical analysis, harmonic analysis for orthogonal polynomials expansions, didactic aspects of mathematical analysis and didactic aspects of geometry. She has published research articles in reputed international journals of of pure and applied mathematics. She serves as both a referee and Editorial Board member for several international journals.

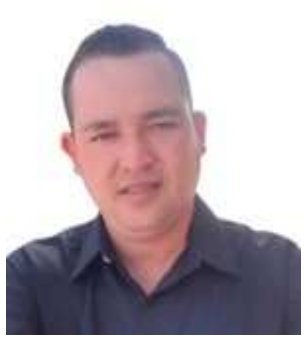

William Ramírez

is Professor and researcher in Faculty of Natural and Exact Sciences at Universidad de la Costa (Colombia), and previously was member of Department of Pure Mathematics at Universidad del Atlántico (Colombia). He received the $\mathrm{MSc}$ degree in

Mathematical Sciences at Universidad del Atlántico. Also, he is a junior researcher recognized by Colciencias (Colombia). His main research interests are: mathematical analysis, number theory and special functions.

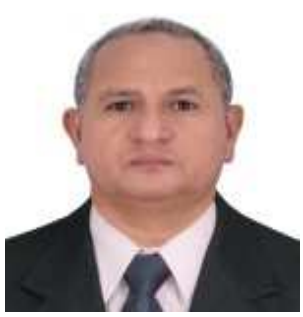

$\begin{array}{cr}\text { Alejandro } & \text { Urieles } \\ \text { is } & \text { Professor }\end{array}$ researcher in Faculty of Basic Sciences-Mathematics Program at Universidad del Atlántico (Colombia), and previously was Assistant Professor in Department of Pure and Applied Mathematics at Universidad Simón Bolívar (Venezuela).

$\mathrm{He}$ received the $\mathrm{PhD}$ degree in Mathematics at Universidad Simón Bolívar. He is a senior researcher recognized by Colciencias (Colombia) and referee of international journals in the frame of pure and applied mathematics. His research has been based mainly on approximation theory and special functions, mathematical analysis and harmonic analysis for orthogonal polynomials expansions. Currently, he coordinates the Hotbed of Research on Approximation Theory and Special Functions at Universidad del Atlántico. 